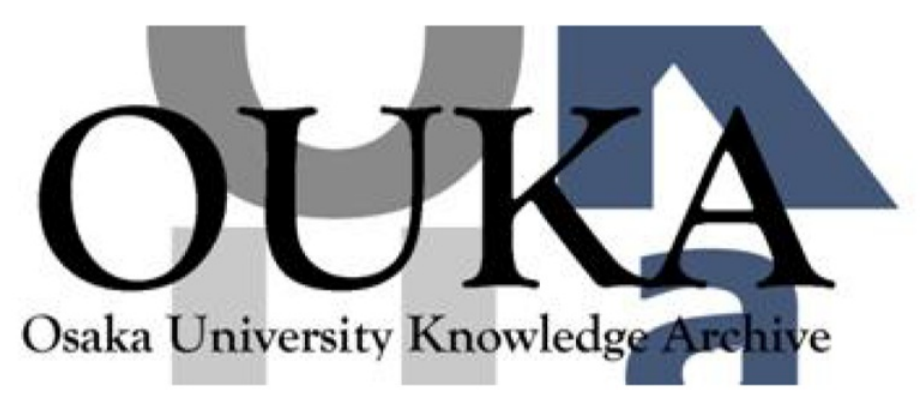

\begin{tabular}{|c|l|}
\hline Title & $\begin{array}{l}\text { Line-focusing of ultrasonics SV wave by } \\
\text { electromagnetic acoustic transducer }\end{array}$ \\
\hline Author(s) & $\begin{array}{l}\text { Ogi, Hirotsugu; Hirao, Masahiko; Ohtani, } \\
\text { Toshihiro }\end{array}$ \\
\hline Citation & $\begin{array}{l}\text { Journal of the Acoustical Society of America. } \\
103(5) \text { p. } 2411-p .2415\end{array}$ \\
\hline Issue Date & $1998-05$ \\
\hline oaire:version & VoR \\
\hline URL & $\begin{array}{l}\text { https://hdl.handle. net/11094/84151 } \\
\text { Copyright 1998 Acoustical Society of America. } \\
\text { This article may be downloaded for personal use } \\
\text { only. Any other use requires prior permission } \\
\text { of the author and the Acoustical Society of } \\
\text { America. }\end{array}$ \\
\hline rights \\
\hline Note & \begin{tabular}{l} 
\\
\hline
\end{tabular} \\
\hline
\end{tabular}

Osaka University Knowledge Archive : OUKA

https://ir. Library. osaka-u. ac. jp/

0saka University 


\title{
Line-focusing of ultrasonic $S V$ wave by electromagnetic acoustic transducer
}

\author{
Hirotsugu Ogia) and Masahiko Hirao \\ Graduate School of Engineering Science, Osaka University, Machikaneyama 1-3, Toyonaka, Osaka 560- \\ 8531, Japan \\ Toshihiro Ohtani \\ Ebara Research Co., Ltd., Fujisawa, Kanagawa 251-8502, Japan
}

(Received 10 July 1997; accepted for publication 27 January 1998)

\begin{abstract}
An electromagnetic acoustic transducer has been developed for line-focusing the shear-vertical $(S V)$ wave in a metal. The EMAT consists of a permanent magnet to supply the bias magnetic field normal to the surface, and a meanderline coil to induce the dynamic field and eddy currents in the surface region of the sample. The meanderline spacing is continuously changed so that the generated $S V$ waves from all segment sources become coherent on the focal line after traveling oblique paths. The printed circuit technique enables the fabrication of such a functionally spaced meanderline coil within $1-\mu \mathrm{m}$ accuracy. The directivity is measured using a half-cylindrical sample of an aluminum alloy, which shows a much sharper radiation pattern of the focusing EMAT than the EMAT having a meanderline coil of constant spacings. This EMAT is then tested through detecting a shallow notch. The scattered signal from the notch is received by the same EMAT, which shows enough strength even for the notch of 0.2-mm depth. () 1998 Acoustical Society of America.
\end{abstract}

[S0001-4966(98)03205-6]

PACS numbers: 43.35.Zc [HEB]

\section{INTRODUCTION}

Electromagnetic acoustic transducers (EMATs) ${ }^{1-5}$ have definite advantages for the noncontact inspection and characterization of electrically conductive materials. They excite and detect the elastic waves through the electromagnetic transduction and they are free from any mechanical coupling, which eliminates the measurement errors associated with the contact transduction. An EMAT consists of permanent magnets (or electromagnets) to supply the bias magnetic field, and the driving coil element to excite the eddy currents and the dynamic fields. Their configuration depends on the mode of elastic wave desired.

An EMAT, however, has two basic disadvantages; the weak transduction efficiency and the broad radiation pattern of elastic waves into a solid. However, the sensitivity of the EMAT has been dramatically improved owing to the recent advances in the electronics and signal processing techniques, the permanent magnet such as the $\mathrm{Nd}-\mathrm{Fe}-\mathrm{B}$ sintered magnet, and the printed circuit techniques to fabricate the printed coils. Especially, the use of an EMAT in the ultrasonic resonant technique ${ }^{6-9}$ overcame the low transduction efficiency by receiving highly and coherently overlapping echoes at a resonant state. On the other hand, the elastic waves generated by an EMAT propagate nearly all possible directions in the sample, which is undesirable for flaw detection purposes. For example, Fig. 1 illustrates the elastic-wave radiation by the EMAT built with a meanderline coil and a permanent magnet. The EMAT generates the logitudinal wave and the shear-vertical $(S V)$ wave propagating obliquely in the sample, and the Rayleigh wave along the surface. They are

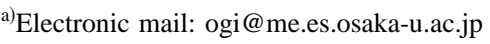

generated at the same time for both sides with the broad directivity patterns and will be reflected not only by flaws but also by the sample edges. It is often difficult to distinguish the flaw signal from others. Moreover, the broad radiation decreases the ultrasonic energy to be concentrated on the target flaw.

This paper presents a technique to sharpen the directivity by line-focusing the $S V$ waves. The major radiation direction by the EMAT in Fig. 1 depends on the driving frequency, the meanderline spacings, and the wave modes. ${ }^{10}$ We choose to focus the $S V$ waves at a fixed frequency by continuously changing the meanderline spacing so that the excited $S V$ waves become coherent on a focal line.

Another technique is possible for focusing the shearhorizontal $(\mathrm{SH})$ wave by the periodic-permanent-magnet EMAT (PPM-EMAT) ${ }^{5}$ which consists of the elongated spiral coil and the periodically arrayed thin permanent magnets. The direction of the $S H$ wave is governed by the spatial period of the magnet array and the frequency. The $S H$ wave can be concentrated on a focal line by changing the magnet thickness. However, this method for focusing the $S H$ wave is impractical for many purposes, because the megahertz-range $S H$ wave needs the magnets thinner than $1 \mathrm{~mm}$, which are intolerable to even a small liftoff. Besides, the present focusing technique is superior at two points. One is the high accuracy for making the meanderline coil. Unlike the permanent magnet case, the spacing of the meanderline coil can be decreased as small as the fabrication accuracy. The recent printed circuit technique makes it possible to produce the meanderline coil of theoretically generated unequal spacing within $1-\mu \mathrm{m}$ accuracy. The second advantage is in the use of the $S V$ wave itself. The $S V$ wave radiated from a line source 


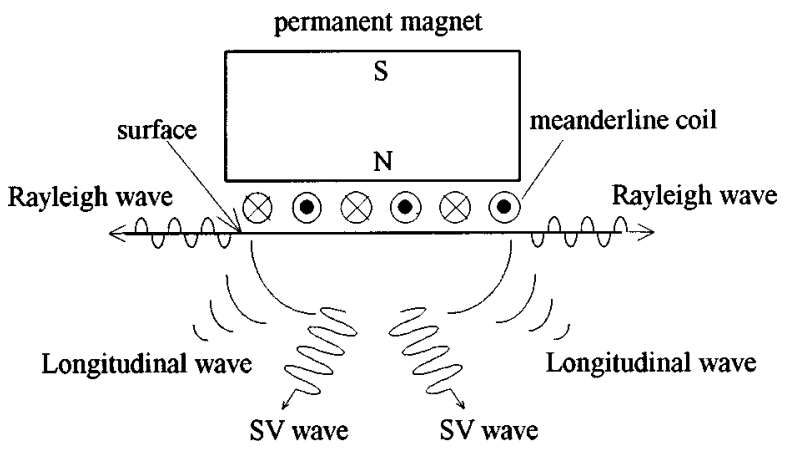

FIG. 1. Typical elastic wave radiation by an EMAT with a permanent magnet and a meanderline coil with equal spacings.

has a sharp directivity, ${ }^{11}$ unlike the broad radiation patterns of other elastic modes.

\section{DESIGN OF THE MEANDERLINE COIL}

We consider a two-dimensional space, where the halfspace of $z>0$ is filled with an electrically conducting material and the $x-y$ plane coincides with the surface (Fig. 2). A meanderline coil and a permanent magnet are located near the surface. When the coil is driven by a current, the shearing forces parallel to the surface arise mainly under the straight parts of the coil due to the Lorentz force, the magnetization force, and the magnetostriction effect. ${ }^{4,5}$ Only the Lorentz force is the possible source in a nonmagnetic metal, but all three contribute to producing the shearing force in a ferromagnetic metal.

When the meanderline coil has a constant spacing $l$, the angle $\theta$ for the major radiation is determined so that the elastic wave originated from each source becomes coherent: ${ }^{10}$

$$
\sin \theta=\frac{c}{2 l f},
$$

where $c$ is the longitudinal or shear wave velocity and $f$ is the frequency of the driving current. On the basis of this relation, we intend to focus the $S V$ wave to a line in the sample using the variable spacing. Figure 3 illustrates the

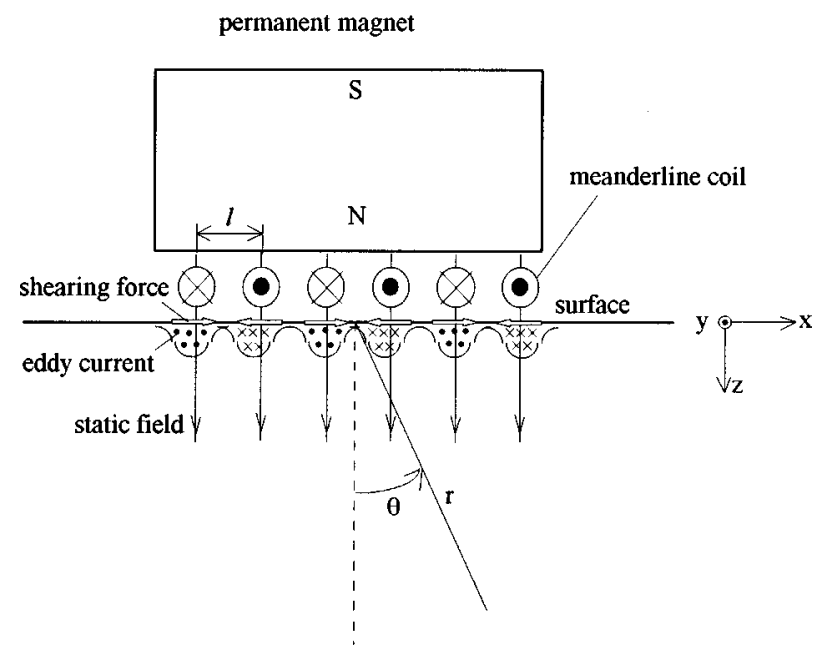

FIG. 2. Two-dimensional field for analyzing the radiation of the EMAT. Only Lorentz force is considered for simplicity.

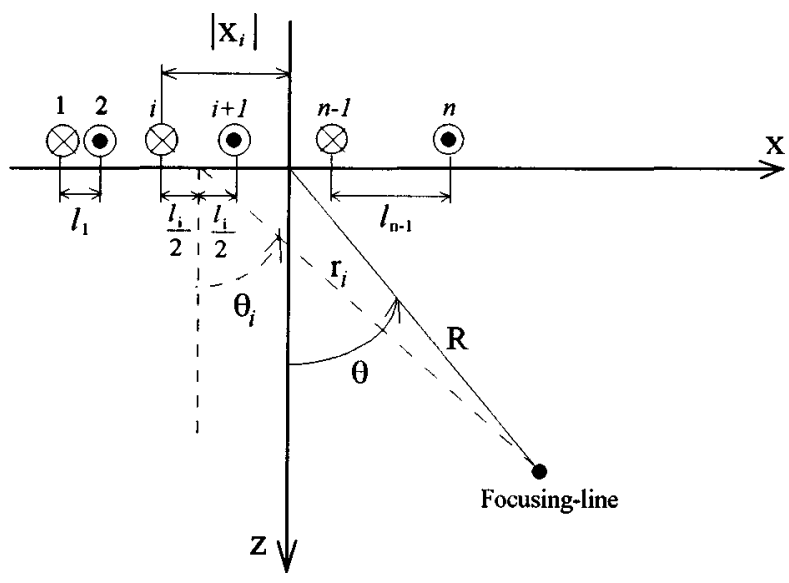

FIG. 3. Determination of the meanderline configuration.

determination of the meanderline spacing. First, we set the frequency, a focal line in the sample, and the edge of the coil at the most left side (i.e., setting $f, \theta, R$, and $x_{1}$ ). The location of $(i+1)$ th line $\left(x_{i+1}\right)$ is determined so that the spacing $l_{i}$ locally satisfies Eq. (1):

$$
\sin \theta_{i}=\frac{c_{S}}{2 l_{i} f}=\frac{\left|R \sin \theta-x_{i}-l_{i} / 2\right|}{\sqrt{(R \cos \theta)^{2}+\left(R \sin \theta-x_{i}-l_{i} / 2\right)^{2}}},
$$

where $c_{S}$ is the shear wave velocity. Equation (2) is solved for $l_{i}$ by Newton's method with known $x_{i}$ and then we can determine the detailed configuration of the meanderline coil. In this study, we set $f=4 \mathrm{MHz}, \theta=40^{\circ}, R=100 \mathrm{~mm}, x_{1}$ $=-30.88 \mathrm{~mm}$, and $n=98$ with $c_{S}=3.2 \mathrm{~km} / \mathrm{s}$. Figure 4 shows the meanderline spacings calculated for the above parameters.

We have fabricated the meanderline coil by printing copper on a polyimide sheet and then etching the copper following the designed pattern. This technique allows us to make a high-impedance coil of an arbitrary geometry with the accuracy of $1 \mu \mathrm{m}$. The liftoff of the sheet coil is only 25 $\mu \mathrm{m}$, improving the weak coupling efficiency of the EMAT.

\section{NUMERICAL SIMULATION OF RADIATION DIRECTIVITY}

A numerical simulation is possible for estimating the radiation pattern of the EMAT. Considering a nonmagnetic

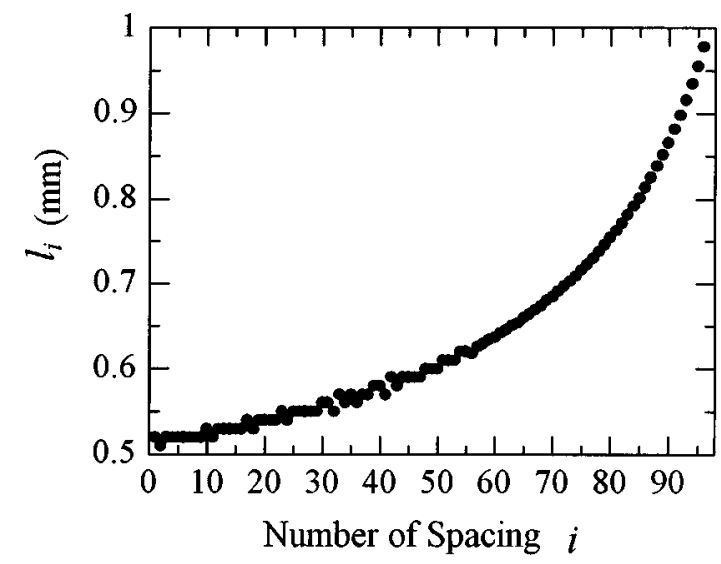

FIG. 4. Variable of the meanderline coil.

Ogi et al.: Line-focusing of ultrasonic $S V$ wave 


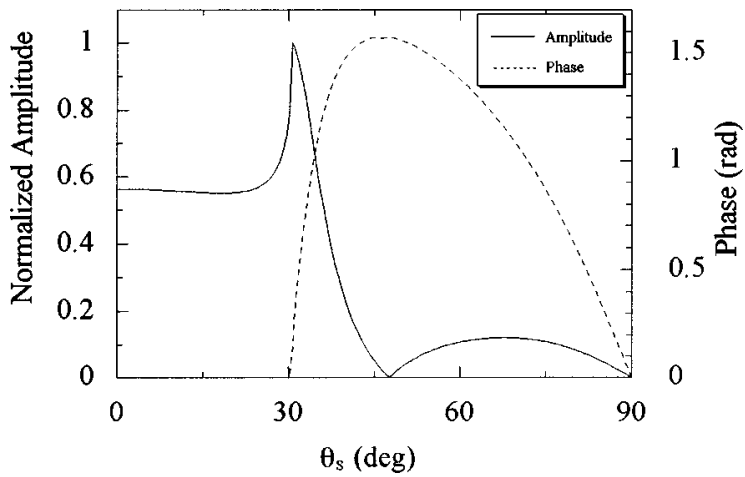

FIG. 5. Directivity of the amplitude and the phase of the $S V$ wave radiated from the single strip source.

metal, the EMAT shown in Fig. 2 excites the shearing Lorentz force in the $x$ direction in the surface region. When the sample material has a good conductivity, the shearing force takes place in the surface skin and can be considered as the traction force on the $x-y$ plane. ${ }^{12}$ Thompson ${ }^{5}$ derived the Fourier transform expression for the periodic traction force and, by taking up the fundamental component, showed that it is proportional to $\cos (\pi x / l)$. We apply this formula to the small part of the coil, assuming the local uniformity of the spacings. On the surface region between the $i$ th and $(i$ $+1)$ th lines, we approximate the traction force by the cosine curve with the characteristic length $l_{i}$ (spatial frequency of $\left.\pi x / l_{i}\right)$.

We then digitize the traction-force distribution to a large number of strip sources at equal intervals to calculate the amplitude and the phase of the $S V$ wave radiated from the EMAT. Each element source oscillates with the strength and phase prescribed by the cosine function. When a strip source, lying along the $y$ axis at $x=0$ and having a very narrow width compared with the wavelength, vibrates along the $x$ axis, the displacement $u_{\theta}$ perpendicular to the propagation direction of the excited $S V$ wave is generated: ${ }^{11}$

$$
\begin{aligned}
& u_{\theta}\left(r, \theta_{S}\right) \\
& =\frac{C}{\sqrt{r}} \frac{(\lambda+2 \mu) \cos 2 \theta_{S} \cos \theta_{S}}{2 \mu \sin 2 \theta_{S} \sin \theta_{L} \cos \theta_{L}+(\lambda+2 \mu) \cos ^{2} 2 \theta_{S}} \\
& \quad \times e^{j\left[(3 / 4) \pi-k_{S} r\right]}, \\
& \cos ^{2} \theta_{L}=1-\left(\frac{\lambda+2 \mu}{\mu}\right) \sin ^{2} \theta_{S},
\end{aligned}
$$

at a far distance $r$ from the source. Here $C$ is a constant, $\lambda$ and $\mu$ are Lame's constants, $k_{S}\left(=\omega / c_{S}\right)$ is the wave number of the $S V$ wave, and $\theta_{S}$ is the propagation direction of the $S V$ wave. Figure 5 shows the normalized amplitude and the relative phase referring to $\theta_{S}=0^{\circ}$ of the $S V$ wave radiated from the single strip source as functions of $\theta_{S}$ for $c_{S}$ $=3.2 \mathrm{~km} / \mathrm{s}, \mu=26 \mathrm{GPa}$, and $\lambda=58 \mathrm{GPa}$. We see that the amplitude directivity has a sharp peak around $\theta_{S}=30^{\circ}$, which corresponds to the critical angle of the longitudinal wave $\left(\theta_{L}=90^{\circ}\right)$, and all the exciting energy is directed toward the shear wave generation. Therefore, it is beneficial to locate the focal line so that all the sources take the angle $\theta_{i}$ centered around this angle, although Eq. (1) must be satisfied. The angle $\theta_{i}$ ranges from $23^{\circ}$ to $51^{\circ}$ with the meanderline coil designed in this study (Fig. 4).

By applying Eq. (3) to all the strip sources and numerically integrating their contributions, ${ }^{13}$ we map the amplitude and the phase of the $S V$ wave in the two-dimensional region. Figure 6 shows the result for the line-focusing EMAT (LFEMAT) with the coil designing of Fig. 4 and, for comparison purposes, the constant-spaced EMAT (CS-EMAT) of $l$ $=0.65 \mathrm{~mm}$. Attenuation is not included. The results are normalized by the maximum amplitude in the LF-EMAT case. We find that the LF-EMAT successfully focuses the $S V$ wave at the position as expected $(x=64.2 \mathrm{~mm}, z=76.6 \mathrm{~mm})$, while the CS-EMAT produces the broad radiation pattern
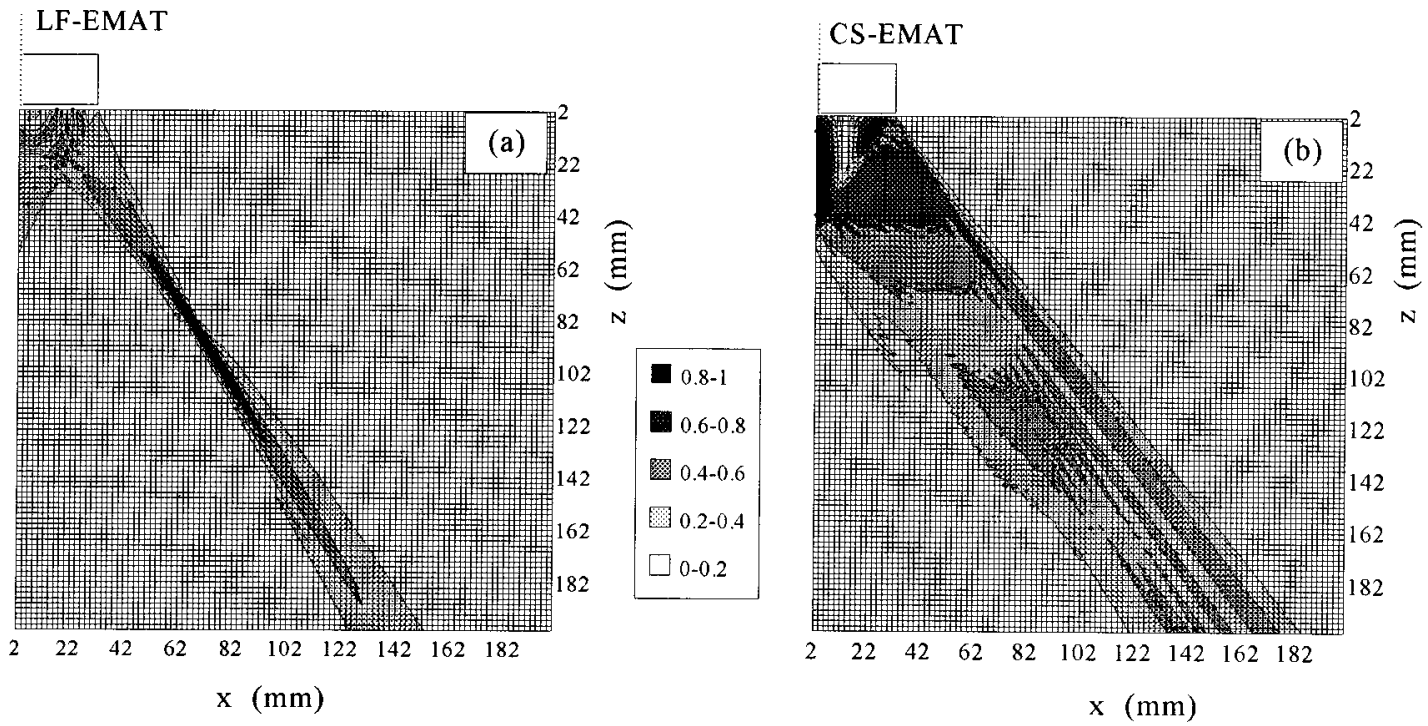

FIG. 6. Simulation of the two-dimensional distribution of the $S V$-wave amplitude radiated by (a) the LF-EMAT and (b) the CS-EMAT. 


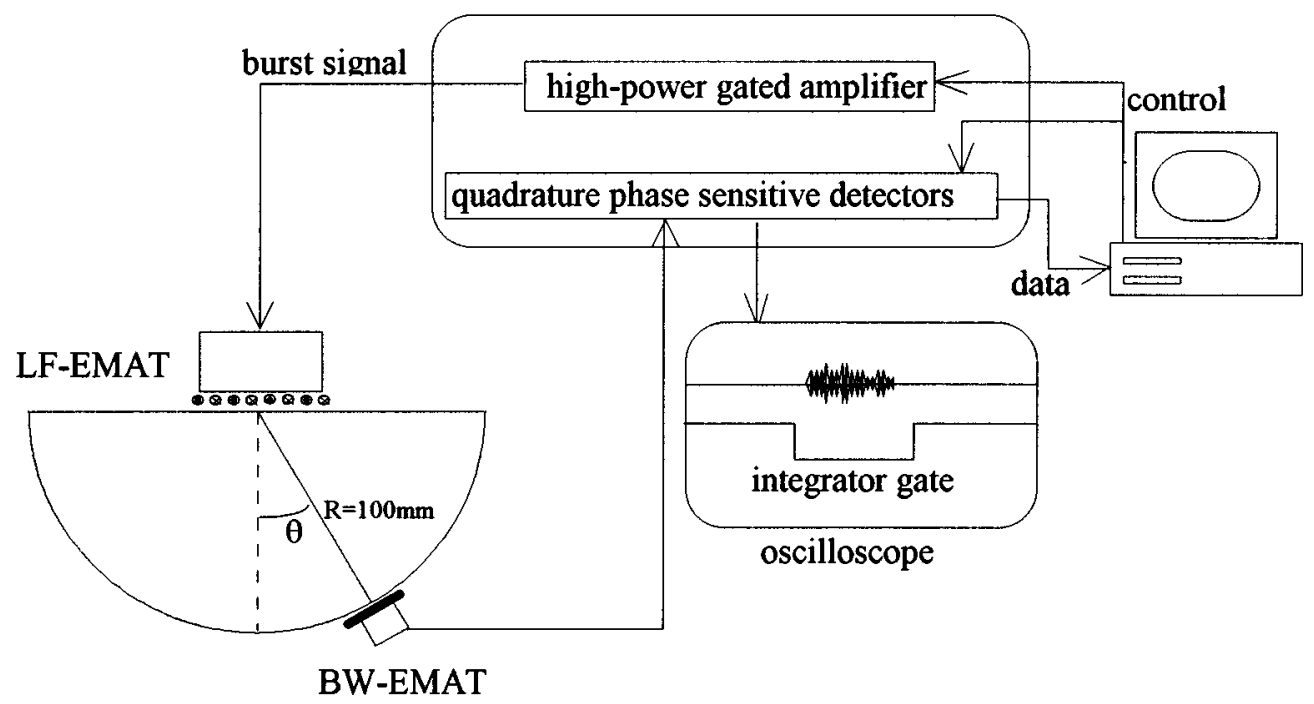

FIG. 7. Measurement setup for the directivity of the $S V$-wave amplitude.

and naturally the peak amplitude is smaller than the LFEMAT case.

\section{MEASUREMENT OF DIRECTIVITY}

Figure 7 sketches the measurement setup for the radiation pattern of the EMATs. We use a half-cylindrical sample of an aluminum alloy (Al2017), whose diameter is $200 \mathrm{~mm}$ and axial length is $100 \mathrm{~mm}$. The EMAT is located on the middle of the flat surface and is driven by high-power burst signals of $8-\mu$ s duration. The $S V$ waves generated by the EMAT are received by the bulk-wave EMAT ${ }^{6,8}$ located on the cylindrical surface. To produce a good spatial resolution for the directivity measurement, the coil face of the bulkwave EMAT is shielded with copper sheets to make a 1-mmwide aperture. The received signal is fed to the quadrature phase detectors to extract the in-phase and out-of-phase outputs relative to the driving signal by analog-integrator gates of $30-\mu$ s width. The amplitude is determined from the root of their squares. The details of electronics are found in the pervious work. ${ }^{6}$

The measurements have been performed for two EMATs, the LF-EMAT and the CS-EMAT $(l=0.65 \mathrm{~mm})$. Figure 8 shows the amplitude distribution as a function of the propagation direction. This includes the results of the numerical simulation, which shows a good agreement with the measurement, indicating the validity for estimating the radiation pattern. It is clear that the LF-EMAT is much superior to the CS-EMAT in the directivity sharpness. We also show the measured directivity pattern when the LF-EMAT is driven at $2.5 \mathrm{MHz}$. As the driving frequency decreases, the amplitude peak moves to larger angle region. This is easily understood from Eq. (1), but the directivity shows the sharpest peak at the designed angle and frequency.

We show the as-received signals at $4 \mathrm{MHz}$ for three angles in Fig. 9. They also indicates the good directivity of the LF-EMAT. The signal from the LF-EMAT at the focusing angle $\left(\theta=40^{\circ}\right)$ is not flat, which is attributed to the transit time difference from the sources. The meanderline coil was about $60 \mathrm{~mm}$ long. The maximum difference occurs between the edge sources and it is estimated to be $12 \mu \mathrm{s}$. In the measurement, we used an $8-\mu$ s burst signal. Therefore, they are partially superimposed and the received signal takes the shape of a larger duration.

\section{DETECTION OF SHALLOW NOTCH}

The LF-EMAT was then used for detecting the shallow notch on the opposite surface of an aluminum block (76-mm hight, 60-mm width, and 500-mm length) introduced by the electrodischarge technique. The notch was $20 \mathrm{~mm}$ long, 1.0 $\mathrm{mm}$ wide, and $0.2 \mathrm{~mm}$ deep. The notch was located at the distance of $111.5 \mathrm{~mm}$ from the edge of the block. The focal line of the EMAT lays on the opposite surface. By moving the EMAT on the upper surface in the length direction, we measure the amplitude of the scattering signal from the notch.

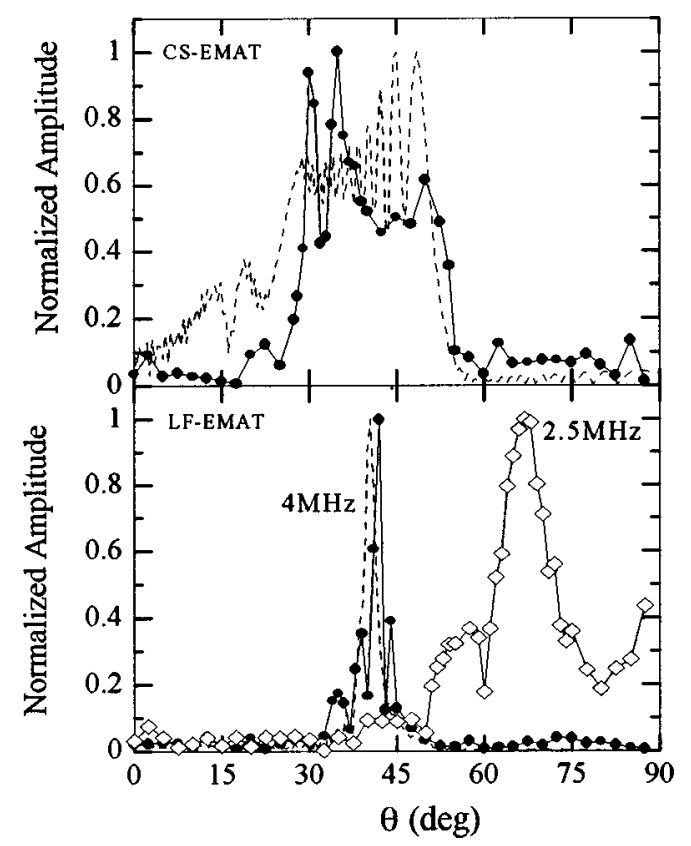

FIG. 8. Measurement and calculation of the directivity of the $S V$-wave amplitude. The solid circles are the data at $4 \mathrm{MHz}$ and the open lozenges at 2.5 $\mathrm{MHz}$. The broken lines show the calculation results at $4 \mathrm{MHz}$. 


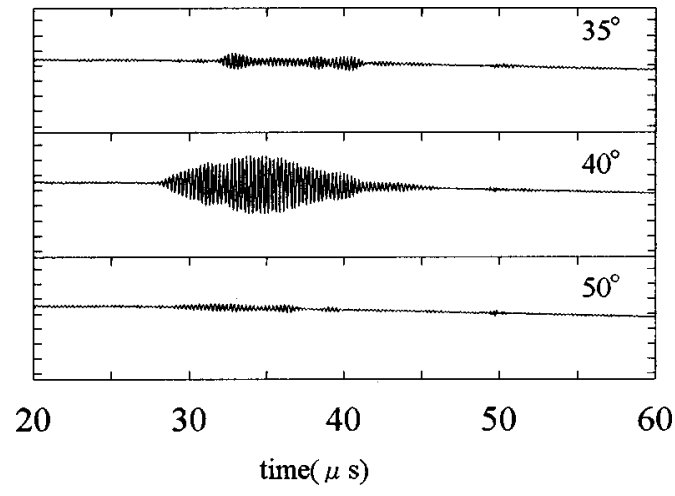

(a) LF-EMAT

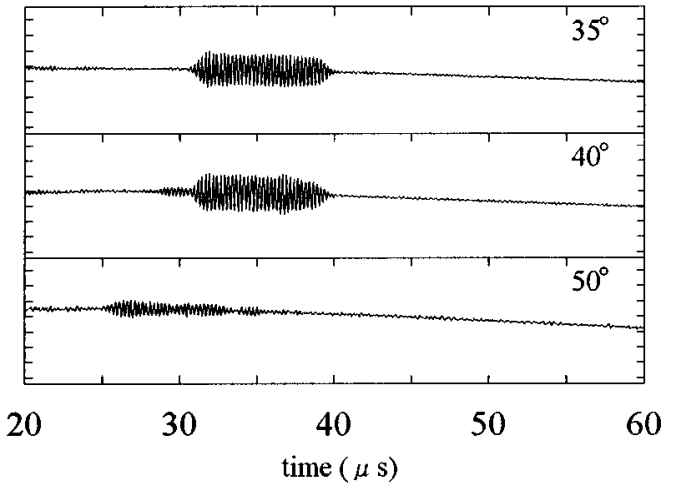

(b) CS-EMAT

FIG. 9. Wave forms received at three angles for (a) the LF-EMAT and (b) the CS-EMAT.

The same EMAT receives the signals. The result is plotted in Fig. 10. If the focal line is away from the notch, the amplitude is as small as the noise level. Approaching the notch, the amplitude increases and, after taking the peak, it suddenly decreases back to the noise level. We show the maximum scattering signal in Fig. 11, where the signal has the flat shape and the duration is comparable to that of the input burst signals of $16 \mu \mathrm{s}$, unlike the previous case in Fig. 9(a). This can be explained as follows. The directivity pattern of the $S V$ wave generated from the single source (Fig. 5) is also applicable to express the efficiency directivity in the receiving stage. The total transduction efficiency is governed by the square of this pattern and the peak around $\theta_{S}=30^{\circ}$ is much more enhanced. Therefore, the coil parts located near

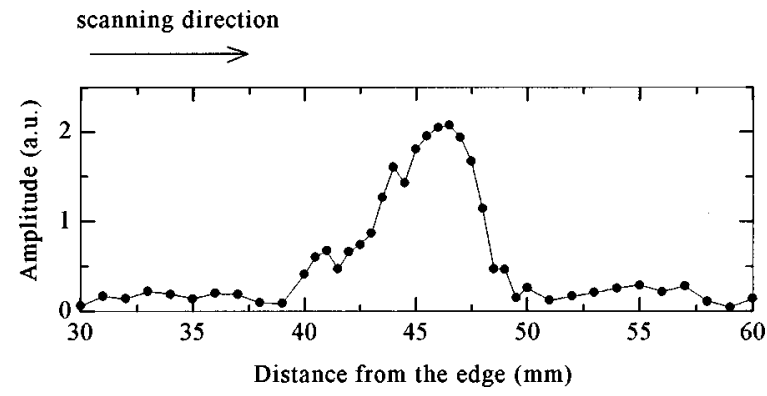

FIG. 10. Amplitude of the scattering echo from the notch detected by the LF-EMAT at $4 \mathrm{MHz}$.

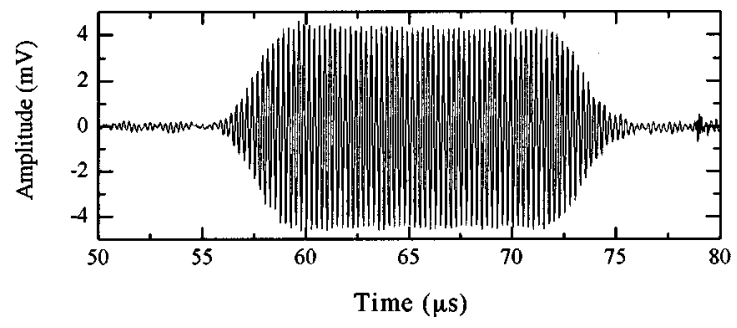

FIG. 11. Scattered echo from the notch of $0.2 \mathrm{~mm}$ deep.

this angle primarily contribute to the received signal. Considering only these parts, the transit time is approximately the same, which makes such a flat signal. We measured the transit time of the received signal through the phase ${ }^{14}$ and obtained $57.8 \mu \mathrm{s}$. The distance between the line source located at $\theta_{S}=33^{\circ}$ and the notch bottom was $91.7 \mathrm{~mm}$, and the transit time of the round trip is then calculated to be $57.3 \mu \mathrm{s}$ with $c_{S}=3.2 \mathrm{~km} / \mathrm{s}$. This agreement supports the above explanation.

\section{CONCLUSIONS}

The line-focusing EMAT has been developed for concentrating the $S V$ wave in a metal by designing the variable spacing of the meanderline coil. Owing to the high accuracy of fabricating the printing coil and the unique radiation pattern of the $S V$ wave, the line-focusing EMAT establishes a sharper directivity pattern. The numerical simulation has been used for predicting the radiation pattern of the EMAT, which showed a good agreement with the measurement. The directivity measurement proved a sharper amplitude peak of the focusing EMAT than the EMAT with an equal-spacing coil. The measurement also indicated that the location of the focal line can be controlled through the frequency, though the directivity is broadened.

The line-focusing EMAT showed a pronounced sensitivity for detecting the shallow notch of 0.2 -mm-depth on the opposite surface of a block. The echo from the notch was large enough and it will be measurable even for smaller notches. The line sources having the angle near $30^{\circ}$ to the focal line give the largest contribution to the flaw detection.

${ }^{1}$ R. B. Thompson, IEEE Trans. Sonics Ultrason. SU-25, 7 (1978).

${ }^{2}$ W. B. Maxfield and C. M. Fortunko, Mater. Eval. 41, 1399 (1983).

${ }^{3}$ K. Kawashima, IEEE Trans. Sonics Ultrason. SU-32, 514 (1985).

${ }^{4}$ A. Wilbrand, Review of Progress in QNDE, edited by D. O. Thompson and D. E. Chimenti (Plenum, New York, 1987), Vol. 7A, p. 671.

${ }^{5}$ R. B. Thompson, Physical Acoustics (Academic, New York, 1990), Vol. 19, p. 157.

${ }^{6}$ M. Hirao, H. Ogi, and H. Fukuoka, Rev. Sci. Instrum. 64, 3198 (1993).

${ }^{7}$ W. L. Johnson, B. A. Auld, and G. A. Alers, Review of Progres in QNDE, edited by D. O. Thompson and D. E. Chimenti (Plenum, New York, 1994), Vol. 13, p. 1603.

${ }^{8}$ H. Ogi, M. Hirao, and T. Honda, J. Acoust. Soc. Am. 98, 458 (1995).

${ }^{9}$ H. Ogi, M. Hirao, and K. Minoura, J. Appl. Phys. 81, 3677 (1997).

${ }^{10}$ T. J. Moran and R. M. Panos, J. Appl. Phys. 47, 2225 (1976).

${ }^{11}$ G. F. Miller and H. Pursey, Proc. R. Soc. London, Ser. A 223, 524 (1954).

${ }^{12}$ M. R. Gaerttner, W. D. Wallace, and B. W. Maxfield, Phys. Rev. 184, 702 (1969).

${ }^{13}$ H. Ogi, M. Hirao, and T. Honda, J. Acoust. Soc. Am. 98, 1191 (1995).

${ }^{14}$ M. Hirao, H. Ogi, and H. Fukuoka, Res. Nondestruct. Eval. 5, 211 (1993). 